\title{
Création du Club de Biochimie du Sperme
}

\section{André ClaverT}

Lors de la session "Plasma séminal" du Congrès de Périconceptologie de Toulouse organisé en mai 1993, Marco Balerna fit un exposé brillant qui démontra que la spermiologie a besoin de nouveaux concepts pour faire progresser les traitements de l'hypofertilité masculine.

Si la fécondation in vitro a permis de mieux comprendre les mécanismes d'interaction entre le spermatozoïde et l'œuf, nous sommes par contre arrivés à un stade où il faut réaborder l'analyse des différentes étapes de la maturation du spermatozoïde car ces étapes conditionnent le déroulement ultime de la fécondation.

En pratique, nous sommes, le plus souvent, confrontrés au problème délicat d'une hypofertilité masculine responsable d'échecs de fécondation in vitro. Ces échecs sont liés, dans bon nombre de cas, au fait que la FIV a été réalisée sans exploration suffisante de la fécondance du sperme et sans tentative thérapeutique.

Ce comportement est lié au fait que nous sommes psychologiquement dans une situation d'impuissance face à cette stérilité masculine. Pourtant en regardant de plus près, il apparait évident que des thérapeutiques existent, mais jusqu'à présent les différentes tentatives se sont révélées décevantes car vraisemblablement mal menées et mal testées par manque de méthodologies fiables.

L'une des étapes de la maturation du spermatozoïde qui paraît être facilement accessible aux techniques de laboratoires et aux thérapeutiques, est le sperme. Or des concepts manquent pour développer des techniques et pour instaurer de nouvelles thérapeutiques.

Les exposés qui ont été fait lors de cette session toulousaine ont montré combien le plasma séminal est complexe, comment l'infection du tractus génital peut perturber sa composition et ses fonctions, comment le spermatozoïde peut être lésé par la peroxydation. De nouvelles pistes ont été évoquées quant au diagnostic de ces perturbations. Il apparaît ainsi logique qu'un traitement bien mené à partir d'un bon diagnostic puisse éviter une perte de fécondance.

Pour faire évoluer les concepts, nous vous proposons de constituer sous l'égide de la SALF un "Club de Biochimie du Sperme" ou toute personne intéressé puisse échanger observations, idées et réflexions.

Nous pourrions procéder en deux temps :

- recensement des sujets de recherche et diffusion des références des publications des différentes équipes,

- organisation d'une réunion thématique annuelle.

Pour augmenter nos contacts avec nos voisins et amis, le français mais également l'anglais pourront être utilisés comme langue d'échange.

Pour tout renseignement complémentaire vous pouvez vous adresser au:

Docteur André CLAVERT. Groupe de recherche en Andrologie - Faculté de Médecine,

11, rue Humann -F67085 Strasbourg. Fax : (33) 88.35.87.29. 